\title{
BMJ Open Combating escalating harms associated with pharmaceutical opioid use in Australia: the POPPY II study protocol
}

\author{
Natasa Gisev, ${ }^{1}$ Sallie-Anne Pearson, ${ }^{2}$ Timothy Dobbins, ${ }^{1}$ David C Currow, ${ }^{3}$ \\ Fiona Blyth, ${ }^{4}$ Sarah Larney, ${ }^{1}$ Adrian Dunlop, ${ }^{5,6}$ Richard P Mattick, ${ }^{1}$ Andrew Wilson, ${ }^{7}$ \\ Louisa Degenhardt ${ }^{1}$
}

To cite: Gisev N, Pearson S-A, Dobbins T, et al. Combating escalating harms associated with pharmaceutical opioid use in Australia: the POPPY II study protocol. BMJ Open 2018:8:e025840. doi:10.1136/ bmjopen-2018-025840

- Prepublication history and additional material for this paper are available online. To view these files, please visit the journal online (http://dx.doi. org/10.1136/bmjopen-2018025840).

Received 4 August 2018 Revised 26 September 2018 Accepted 26 September 2018

Check for updates

(c) Author(s) (or their employer(s)) 2018. Re-use permitted under CC BY-NC. No commercial re-use. See rights and permissions. Published by BMJ.

For numbered affiliations see end of article.

Correspondence to

Dr Natasa Gisev;

n.gisev@unsw.edu.au

\section{ABSTRACT}

Introduction 0pioid prescribing has increased 15-fold in Australia in the past two decades, alongside increases in a range of opioid-related harms such as opioid dependence and overdose. However, despite concerns about increasing opioid use, extramedical use and harms, there is a lack of population-level evidence about the drivers of long-term prescribed opioid use, dependence, overdose and other harms.

Methods and analysis We will form a cohort of all adult residents in New South Wales (NSW), Australia, who initiated prescribed opioids from 2002 using Pharmaceutical Benefits Scheme dispensing records. This cohort will be linked to a wide range of other datasets containing information on sociodemographic and clinical characteristics, health service use and adverse outcomes (eg, opioid dependence and non-fatal and fatal overdose). Analyses will initially examine patterns and predictors of prescribed opioid use and then apply regression and survival analysis to quantify the risks and risk factors of adverse outcomes associated with prescribed opioid use. Ethics and dissemination This study has received full ethical approval from the Australian Institute of Health and Welfare Ethics Committee, the NSW Population and Health Services Research Committee and the ACT Health Human Research Ethics Committee. This will be the largest postmarketing surveillance study of prescribed opioids undertaken in Australia, linking exposure and outcomes and examining risk factors for adverse outcomes of prescribed opioids. As such, this work has important translational promise, with direct relevance to regulatory authorities and agencies worldwide. Project findings will be disseminated at scientific conferences and in peer-reviewed journals. We will also conduct targeted dissemination with policy makers, professional bodies and peak bodies in the pain, medicine and addiction fields through stakeholder workshops and advisory groups. Results will be reported in accordance with the REporting of studies Conducted using Observational Routinely collected Data (RECORD) Statement.

\section{INTRODUCTION}

Australia has seen dramatic shifts in the rate of opioid prescribing in the last two decades, including changes in the types of opioids prescribed. Almost 15 million opioid

\section{Strengths and limitations of this study}

- This will be the largest postmarketing surveillance study of prescribed opioids undertaken in Australia, linking exposure and outcomes and examining risk factors for adverse outcomes of prescribed opioids.

- The size of the cohort permits in-depth analyses and comparisons of outcomes for different patient groups, types of opioids prescribed and across different time periods.

- Estimating opioid exposure in pharmaceutical claims data is a complex undertaking and will be challenging.

- Self-reported health and outcome measures are not available from linked administrative data sources and will therefore be assessed using validated tools and proxy measures.

prescriptions were dispensed in Australia in $2015^{1}$ and prescribing increased 15 -fold between 1992 and 2012. ${ }^{2}$ Originally registered to manage cancer and acute pain, since 1999, opioids have been approved to treat an increasing number of chronic non-cancer pain (CNCP) conditions, ${ }^{3}$ despite a lack of evidence of long-term effectiveness. ${ }^{4-6}$

There has also been a shift in the type of opioids prescribed. In 1990, $90 \%$ of opioid dispensings were for so-called weak opioids and $96 \%$ were short-acting opioids. ${ }^{3}$ By 2011 , $40 \%$ of dispensings were for strong opioid and $50 \%$ for long-acting opioids. ${ }^{3}$ In parallel to escalating use, there is increasing extramedical opioid use, injection, opioid-related hospitalisation, opioid dependence and overdose. $^{7-10}$

Oxycodone has received considerable attention due to associated harms. ${ }^{11-13}$ Its prescription has played a significant part in the US opioid epidemic. ${ }^{14}$ Australian evidence suggests patients at higher risk of adverse opioid outcomes may have a higher likelihood of being prescribed oxycodone $\mathrm{e}^{15}$; oxycodone is by far the most commonly 
misused prescription opioid. ${ }^{16}{ }^{17}$ This is concerning given oxycodone utilisation increased 12-fold in Australia since 2000 , now accounting for $34 \%$ of all pharmaceutical opioid use. ${ }^{318}$

Oxycodone is not the only concern. The prescribed opioid market is highly dynamic. The Pharmaceutical Benefits Advisory Committee (PBAC)-Australia's expert advisory group that makes recommendations to the Federal government about medicine listings on the national Pharmaceutical Benefits Scheme (PBS) receives three to six applications for opioid listings annually. Between 2000 and 2016, the PBAC has approved 37 new opioids, formulations, strengths and indications, including formulations intended to deter extramedical use and diversion and reduce harms. Yet, there is little evidence to inform possible PBAC decisions such as limiting the number of subsidised opioids, dose ranges, duration of therapy and formulations. There are challenges for regulatory agencies and third-party payers in this area with regards to balancing treatment access for pain and the risks of inappropriate prescribing.

\section{Pharmaceutical opioid use is increasing rapidly}

CNCP, cancer pain and injuries are major causes of disease burden. ${ }^{19}$ Cancer accounts for $17 \%$ of the total burden of disease (measured by both disability and death), CNCP for $13 \%$ of burden and injuries for $8 \% .{ }^{19}$ The burden associated with these conditions, especially CNCP, has increased in many high-income countries with ageing populations. ${ }^{19}$ As the Australian population ages, these burdens will continue to increase. In direct line with this increasing health burden, as documented above, utilisation of prescription and non-prescription opioids has also dramatically increased.

There are many potential risks of this escalating trend in opioid utilisation. The US example is sobering: it is experiencing an unprecedented epidemic of opioid use and dependence, with substantial increases in opioid deaths, ${ }^{20}$ initiation of heroin use in people prescribed opioids $^{21}$ and enormous strain on the health system. The problems observed with opioid use in the USA have been driven substantially by widespread prescribing of long-acting and strong opioids, such as oxycodone and fentanyl..$^{22}{ }^{23}$ In 1995, the US Food and Drug Administration (FDA) approved the use of controlled-release oxycodone (CRO) for 'initial treatment for moderate to severe non-cancer pain'. It was heavily marketed for use in CNCP, with unsubstantiated claims that it minimised risks of extramedical use and dependence. ${ }^{14}$ Ten years later, an opioid epidemic was well underway. ${ }^{11}{ }^{13}$ Fatal opioid overdoses now outnumber road accident deaths in the USA ${ }^{24}$ opioids are among the top five causes of hospitalisation $^{25}$ and decreases in life expectancy among white non-Hispanic people in the USA have been attributed to escalating fatal opioid overdoses. ${ }^{26}$

In 2016, the US government provided an additional US\$1.1 billion in funding to expand opioid dependence treatment, and more conservative guidelines for opioid prescribing from the US Centers for Disease Control were introduced. ${ }^{27}$ Other strategies that have been implemented include the development of so-called abuse-deterrent formulations of opioids intended to be less attractive for diversion or tampering. ${ }^{28}$ However, questions remain as to the potential for these formulations to achieve substantial decreases in extramedical use, diversion and harms. ${ }^{29}$

Despite the differences in Australia's healthcare system, there is strong evidence that we are heading in the same direction. In Australia, long-acting formulations are recommended as first-line opioid treatments of $\mathrm{CNCP}^{30}$ which is estimated to affect one in five Australians. ${ }^{31}$ There is considerable concern about the appropriateness of long-term opioid prescribing for $\mathrm{CNCP}^{32}$ and we are seeing increases in opioid-related hospital admissions, dependence and overdose $\mathrm{e}^{7-1033}$; pharmaceutical opioids now cause over $70 \%$ of opioid overdose deaths in Australia. ${ }^{33}$ It is therefore imperative to quantify the risks associated with different opioids and identify those most at risk of harm, in order to prevent and reduce further harms.

We have a limited understanding of the patterns of utilisation and extramedical use of different opioids in Australia

Work to date in Australia and the USA has lacked the capacity to shed light on the magnitude of risks faced by people prescribed different opioids and individual risks for adverse outcomes. For example, through analysis of sales data, we found greater strong opioid utilisation in socioeconomically disadvantaged areas, ${ }^{18}$ but these data do not inform us about individual-level utilisation, nor permit any understanding of outcomes of this higher utilisation. We have documented increases in pharmaceutical overdose deaths, ${ }^{8-10}$ with oxycodone featuring prominently and fentanyl increasing, ${ }^{8}$ but this tells us very little about risk factors for such deaths, the trajectory of opioid utilisation leading to death and the risks for other adverse outcomes of opioids (including other causes of death).

Australian prescribing guidelines recommend oxycodone as a first-line opioid for use in CNCP and also in acute pain when simple analgesics are not effective. ${ }^{30}$ Our previous research has shown $73 \%$ of people initiating strong opioids in Australia are now dispensed oxycodone, ${ }^{34}$ with oxycodone much more likely to be used without prior trial of less potent or non-opioid analgesics. ${ }^{34}$ Furthermore, people with acute and CNCP pain are more likely to be given oxycodone than those with cancer pain. ${ }^{34}$ Fentanyl and buprenorphine are also commonly used in CNCP. ${ }^{15} 3536$ Without linkage to outcome data, we cannot determine what proportion of this prescribing is appropriate nor understand the relationship of prescription to harms, as dispensing data alone does not provide information about clinical characteristics of the patients nor indicate risk of adverse outcomes.

In a cohort of people with CNCP taking opioids $(\mathrm{n}=1500),{ }^{15}$ we found that people taking oxycodone (vs 
other opioids) had higher risks of opioid dependence, aberrant opioid use and other opioid harm. ${ }^{15}$ We also found those prescribed oxycodone were more likely to be: taking higher doses, younger and have mental health issues and substance use disorders. ${ }^{15}$ It is unclear whether this cohort is representative of all patients with CNCP, and cross-sectional analysis precludes any capacity to infer causality. The risks faced by patients initially prescribed opioids for other indications remains unknown.

In summary, each of these findings shed light on concerning features of Australian opioid prescribing that underpin the rationale for this study. Their limitations mean that more information is needed to understand who faces these risks and whether there are different risks for different opioids.

\section{We need to identify patients at highest risk for adverse opioid outcomes}

A range of patient characteristics has been associated with adverse outcomes, for example, overdose mortality, including younger age, male gender, lower socioeconomic status and mental health comorbidity. ${ }^{37-40}$ The term 'adverse selection' has been used to describe this apparent contradiction, whereby the likelihood of a patient receiving opioid therapy increases as the number of risk factors for adverse outcomes increases. ${ }^{40}$ However, much of this work is derived from cross-sectional or retrospective analyses of highly selected patient samples (typically those with $\mathrm{CNCP}$ ), with limited capacity to understand risks of less selected patients using opioids for other reasons (eg, cancer and acute pain).

\section{We need to move from signal detection of harms to quantification of harm}

Since 1961, the WHO Programme for International Drug Monitoring has collated reports of adverse drug reactions (ADRs). ${ }^{41}$ There is increasing recognition that relatively passive systems of ADR detection fall far short of what is needed to understand the magnitude of medicines' risks. ${ }^{42}$ Contemporary postmarketing surveillance (PMS) quantifies the outcomes associated with specific medicine exposures. ${ }^{42}$ Active PMS is increasingly required: the US FDA has dramatically increased its requirements, judging earlier requirements inadequate. ${ }^{43}$

The International Association for the Study of Pain (IASP) Position Statement on Opioids states 'IASP also strongly advocates for continued research to identify ways to minimise opioid risk...' showing the importance of robust risk assessment frameworks. ${ }^{44}$ We have a unique opportunity to undertake such work, quantifying the extent and nature of opioid risk in Australia. We will apply a consistent framework of risk assessment to the entire range of prescribed opioids, with long-registered opioids assessed alongside more recently registered opioids. The contrast with older opioids (eg, morphine) is important because few requirements to document risks existed at the time of approval. We have a particular focus on strong and long-acting opioids.

\section{AIMS}

We will form a population-based cohort of adult residents in New South Wales (NSW), Australia, who initiated prescribed opioids from 2002, with linkage to a range of datasets providing rich information on sociodemographic and clinical characteristics, health service use and adverse outcomes. Our study will meet three aims:

1. Identify patterns of utilisation of different opioids, including indications of non-adherent or aberrant utilisation and establish predictors of different patterns of opioid use.

2. Quantify the risks of adverse outcomes of prescribed opioids and establish predictors of such outcomes, including indicators for prescription, comorbid mental health and physical problems, sociodemographic characteristics, patterns of other health service use and other medicines use.

3. Examine the population-level impact of changes in regulation and subsidy of opioids on patterns of utilisation and risks of adverse outcomes.

\section{METHODS AND ANALYSIS \\ Setting}

Australia has a publicly funded universal healthcare system entitling all Australian citizens and permanent residents to a range of subsidised health services. This includes free treatment in public hospitals (funded jointly by Commonwealth and State/Territory governments), subsidised outpatient services including consultations with medical and selected healthcare professionals (funded by the Commonwealth's Medicare Benefits Scheme) and medicines prescribed in the community and private hospitals (funded by the PBS) ${ }^{45}$ Medicines prescribed to public hospital inpatients are covered primarily by hospital budgets.

\section{Cohort definition}

The cohort will comprise all adult NSW residents who initiated a new opioid dispensing episode from 2002 to most recently available using PBS dispensing records. A new episode will be defined as the first time we observe a PBS record for a prescribed opioid after a period of at least 90 days with no opioid dispensing records. PBS-listed opioid analgesics include: oxycodone, fentanyl, codeine, dextropropoxyphene, hydromorphone, morphine, pethidine, tapentadol, tramadol, buprenorphine and methadone. The complete list of all Anatomical Therapeutic Chemical $^{46}$ and PBS item codes that will be used to extract the cohort is included in online supplementary appendix A. People initiating methadone or buprenorphine for opioid dependence are not included. Private prescriptions (ie, those in which the patient pays the full cost themselves, with no government subsidy) are not included, but these form a very small proportion of overall opioid prescriptions. ${ }^{47}$

This study is informed by our earlier work examining patterns of opioid use and access. ${ }^{34-50}$ We have recently 
Table 1 Description of datasets to be linked*

\section{Dataset name and year of first record}

Pharmaceutical Benefits Scheme (PBS, 2002)

Medicare Benefits Scheme
(MBS, 2002)

$\begin{aligned} & \text { Australian Cancer Database } \\ & \text { (ACD, 1982) }\end{aligned}$
National Death Index (NDI,
2002)
NSW Admitted Patient Data
Collection (NSW APDC, 2001);
ACT Admitted Patient Care
(ACT APC, 2004)

NSW Emergency Department Data Collection (NSW EDDC, 2005); ACT Emergency Department Data Collection (ACT EDDC, 2005)

Pharmaceutical Drugs of
Addiction System (PHDAS,
1985) Changed to Electronic
Recording and Reporting of
Controlled Drugs (ERRCD) in
September 2016.

Mental Health Ambulatory Collection (MH-AMB, 2001)

\section{Description of dataset}

Records for all PBS-listed medicines for which the Commonwealth pays a subsidy (2002-2012). After 2012, all PBS dispensings are included.

Claims for all medical and hospital services subsidised by the Commonwealth including doctor visits, pathology tests and imaging.

All notifications of primary malignant neoplasms.

Death registrations and causes of death.

\section{Purpose of dataset}

To identify the cohort and the types of opioids and other medicines prescribed.

\section{Key variables of interest}

PBS-item number, date of prescribing and dispensing, patient copayment, cost to government and provider location.

To identify the use of medical and hospital services.

MBS-item number, date of service, schedule fee, provider charge, benefit paid, patient copayment and provider location.

To identify individuals treated with opioids for cancer pain.

Date of diagnosis, topography and morphology codes and degree of spread.

To calculate mortality rates for the cohort and censor individuals.

Census of all inpatient episodes in all NSW/ACT public and private hospitals, public multi-purpose services and private day procedure centres.

All visits to participating emergency departments in NSW/ACT.

To identify harms and risks associated with prescribed opioids.

To identify harms and risks associated with prescribed opioids.

Date of death, underlying and contributing causes of death.

Dates of admission, separation and procedures, diagnostic and procedure codes, admission costs, separation mode, hospital type and hospital location.

Dates of presentation and separation, referral source, arrival mode, visit type, triage, diagnosis and separation mode.

\section{Opioid substitution therapy} (methadone/buprenorphine) treatment episodes in NSW.
Treatment entry and exit dates and type of medicine authorised.
To identify individuals with a history of opioid dependence and who subsequently are prescribed opioids. We will also use this as an outcome, examining risk of treatment for iatrogenic opioid dependence.

To identify individuals with mental health disorders and their treatment patterns.

\section{Records on the assessment,} treatment, rehabilitation or care of non-admitted mental health patients in NSW.

${ }^{*}$ Data will be provided from the year indicated. Most collections hold patient demographics including age, sex, location of residence mapped to the socioeconomic ${ }^{80}$ and remoteness classifications. ${ }^{81}$

ACT, Australian Capital Territory; NSW, New South Wales

validated the PBS dataset as a data source for examining population-level opioid utilisation across Australia. ${ }^{47} \mathrm{We}$ have also completed a number of sensitivity analyses to examine the effect of varying the opioid-free window used to identify people initiating opioids, ${ }^{34}{ }^{50}$ demonstrating this is a feasible and acceptable method of defining an incident cohort of persons initiating opioids.

\section{Datasets and linkage}

We will link to 10 Commonwealth and NSW/Australian Capital Territory (ACT) collections (table 1). ACT data collections are included to increase capture of service use by our cohort that may occur in that jurisdiction
(ACT is situated within NSW).$^{51}$ The Australian Institute of Health and Welfare (AIHW) will undertake the data linkage in conjunction with the Centre for Health Record Linkage (CHeReL), who will undertake the linkage of the NSW/ACT collections once the AIHW has established the cohort. AIHW and the CHeReL have track records of quality record linkage and privacy-preserving procedures. Files containing identifying information will be linked using probabilistic record linkage techniques. Coded (non-personally identifiable) matched files will be forwarded to the Secure Unified Research Environment (SURE) facility for access by our team. We will use a 
range of pharmacoepidemiological techniques, measurement methods and analyses, summarised across the three overall aims below.

\section{Patient and public involvement}

Patients were not involved in the design of the study. As described in our dissemination activities, results from this study will be disseminated through one-page summaries distributed to pain clinics, consumer groups and peak bodies. Key findings will also be disseminated via media releases to encourage discussion of findings in a wide variety of news and information outlets.

\section{Analytical plan}

Aim 1: identify patterns of opioid utilisation and establish

predictors of those trajectories

\section{Defining opioid exposure}

The 11 PBS-listed opioid analgesics vary in their strength, potency, formulation, duration of action and route of administration. PBS records do not contain information on prescribed daily dose or the number of days of supply. Therefore, we will estimate the standard coverage days (SCDs) - the median time to resupply of each opioid. We will define a break in opioid treatment as a gap of three SCDs from the date of the last dispensing, consistent with our definition of new treatment. When a treatment break is observed, we will calculate duration of a treatment episode as time from the first prescription to the last prescription plus one SCD. To assess duration of opioid therapy, we will use Kaplan-Meier methods. Concomitant opioid use will be defined as any overlap in SCDs for the same or different opioids.

We will estimate the average daily opioid dose per prescription as the quantity of opioids dispensed multiplied by their strength (in $\mathrm{mg}$ ) and divided by the SCD for that opioid. We will calculate total daily opioid exposure using oral morphine equivalent (OME) milligrams using conversion factors that have been previously developed and synthesised by our group. ${ }^{52}$ This allows for estimates of total opioid exposure to be presented in a common metric for each individual on each day.

Many people will be using multiple strengths of an opioid, or more than one opioid on a given day, particularly for more severe cancer pain and CNCP, although the clinical justification for multiple opioids is absent. ${ }^{32}$ Our definitions of opioid exposure will account for the range of opioids available and their likely concomitant use. We will also move beyond simplistic exposure measures (yes/no) to models that more closely reflect real-world medicine use, recognising that medicines exposure changes over time. Analysing opioid exposure as a time-dependent variable provides unbiased risk estimates of drug-outcome associations. Sensitivity analyses will determine the impact of varying these definitions.

\section{Patterns of opioid use}

Patterns of opioid use will be operationalised in several different ways:

a. Median duration of opioid treatment: defined as the time from the first opioid dispensing record to the last dispensing record plus 30 days. These estimates can also detail different courses of opioid therapy by accounting for breaks in treatment of more than 60 days.

b. Dose escalation: we will estimate the average daily dose of each opioid prescription dispensed using daily OME $\mathrm{mg}^{52}$; this gives us the capacity to track opioid utilisation across a patient's use of different opioids or opioid formulations within a given treatment episode. We will calculate the changes in average daily doses by prescription and report the number of patients in whom doses are increasing, and by what level, over time.

c. Concomitant use of opioids and other medicines: we will investigate the concomitant use of multiple opioids, in addition to the use of opioids with other prescribed medicines, such as benzodiazepines, antidepressants and antipsychotics. Concomitant use will generally be defined as the observation of at least two dispensing records from different medicines within a specific timeframe of each other. Rules will vary according to the medicines of interest. Furthermore, we will identify individuals at risk of potentially harmful drug-drug interactions deemed to be clinically relevant in the literature and common drug information resources (eg, opioids and benzodiazepines).$^{53-55}$ These will be examined using a previously published approach, ${ }^{56}$ overall,

Table 2 Operationalisation of indications

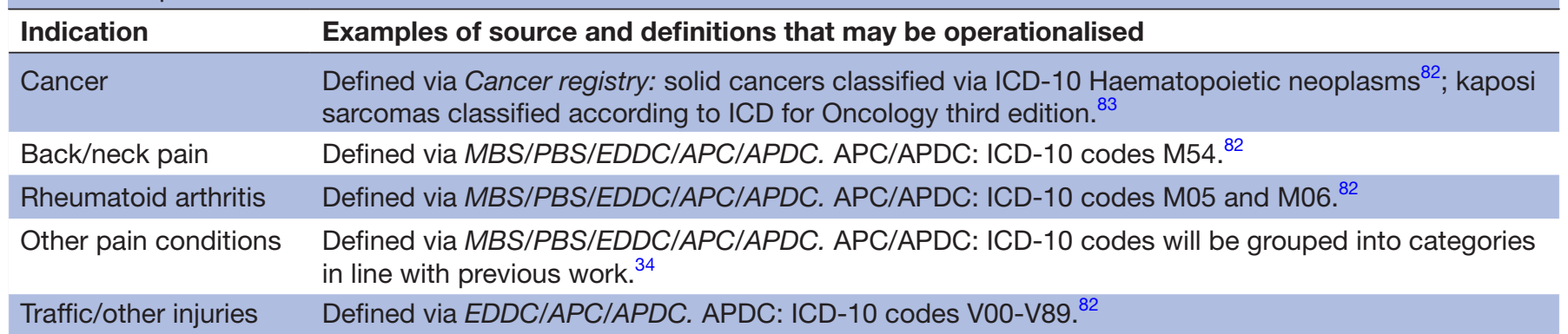

APC, Australian Capital Territory Admitted Patient Care; APDC, New South Wales Admitted Patients Data Collection; EDDC, Australian Capital Territory and New South Wales Emergency Department Data Collections; ICD-10, International Statistical Classification of Diseases and Related Health Problems, 10th Revision; MBS, Medical Benefits Scheme; PBS, Pharmaceutical Benefits Scheme. 
Table 3 Operationalisation of covariates from available data sources

\begin{tabular}{|c|c|}
\hline Joval & nd examples of definitions that may be operationalised. \\
\hline ociodemographics & PBS: age, sex, area of residence, beneficiary status (concessional, general and repatriation). \\
\hline rescription medicine use & PBS: history of other medicine use, specified by drug class, for example, benzodiazepines. \\
\hline Exte & $\begin{array}{l}\text { APC/APDC: application of the Charlson comorbidity index }{ }^{84} ; \text { PBS: application of the RxRisk } \\
\text { tool. }^{85} 86\end{array}$ \\
\hline len & $\begin{array}{l}M H-A M B, M B S, P B S, E D D C \text { and } A P C / A P D C \text { : treatn } \\
\text { mental healthcare plans, outpatient care or hospital }\end{array}$ \\
\hline listory of & $E D D C$ and $A P C / A P D C$ : prior visit for attempted suicide. \\
\hline $\begin{array}{l}\text { istory of substance use } \\
\text { roblems }\end{array}$ & $\begin{array}{l}\text { HDAS: opioid dependence treatment; } M H-A M B \text { : treatment where substance } \\
\text { agged; EDDC/APC/APDC: prior alcohol or illicit drug-related admissions. }\end{array}$ \\
\hline
\end{tabular}

APC, Australian Capital Territory Admitted Patient Care; APDC, New South Wales Admitted Patients Data Collection; EDDC, Australian Capital Territory and New South Wales Emergency Department Data Collections; MBS, Medicare Benefits Scheme; MH-AMB, Mental Health Ambulatory Collection; NDI, National Deaths Index; PBS, Pharmaceutical Benefits Scheme; PHDAS, Pharmaceutical Drugs of Addiction System.

and for specific population subgroups such as older adults.

\section{Extramedical opioid use}

Extramedical opioid use will be defined and operationalised from existing literature ${ }^{57}$ We will report rates of:

a. Excess dosing: defined as average daily dosing outside guideline recommendations. ${ }^{58-60}$

b. Concomitant opioid use: as described above. ${ }^{5961}$

c. Doctor shopping: prescriptions by multiple doctors at medical different practices, dispensed within a specific timeframe. $^{62-71}$

d. Accelerated prescription refill: repeated dispensing of opioids earlier than the time when the dispensing episode is expected to be complete. ${ }^{59} 6072-75$ This will be based on the medication possession ratio and refill compliance.

\section{Defining patient groups}

Table 2 lists examples of patient groups (this is not exhaustive). As per our outcomes of interest, our definitions will be based on existing expert recommendations, ${ }^{34}{ }^{35}$ consultation with data custodians and with clinical experts from our team of investigators and externally.

\section{Key covariates}

We will define a broad range of important clinical and demographic covariates across the linked datasets (see examples in table 3), ensuring we cover domains identified by pain experts as key in analyses of opioid outcomes. ${ }^{36}$ These will include definitions of key patient groups as defined in table 2 (eg, cancer). These will be initially defined at the time of the index opioid prescription. For the survival analyses of risk factors for adverse opioid outcomes, these variables will be considered as time dynamic variables.

Aim 2: quantify risks of adverse opioid-related outcomes and establish predictors of outcomes

provides examples of our approach to identify indicators of opioid-related adverse outcomes. We will use definitions of adverse outcomes consistent with expert recommendations. ${ }^{345}$ In cases where none exist, we will develop definitions in consultation with data custodians and clinical experts from our team of investigators and externally.

We will calculate the event rate (per 1000 days of use) for each adverse outcome examined (see examples in table 4) for each of the opioids by age-group and sex, with CIs derived from a Poisson or negative binomial distribution as appropriate. We will use survival analytic

\begin{tabular}{ll}
\hline Table 4 Operationalisation of example outcomes \\
\hline Example outcomes & Examples of source and definitions that may be operationalised \\
\hline Opioid use disorders & APC/APDC: ICD-10 opioid use disorder (F11 codes) $)^{82}$; EDDC; and PHDAS. \\
Opioid dependence treatment & PHDAS: entry into opioid substitution therapy. \\
Falls & APC/APDC: ICD-10 slipping, tripping, stumbling and falls (W00-W19). ${ }^{82}$ \\
Non-fatal opioid overdose & APC/APDC: ICD-10 opioid poisoning (X42, X44 with T codes). ${ }^{82}$ \\
Accidental opioid-induced death & $\begin{array}{l}\text { NDI: ICD-10 codes for opioid use disorders or poisoning (F11; F19 with F11; F19, X42, X44 } \\
\text { with T codes for opioids). }{ }^{82}\end{array}$ \\
\hline
\end{tabular}

APC, Australian Capital Territory Admitted Patient Care; APDC, New South Wales Admitted Patients Data Collection; EDDC, Australian Capital Territory and New South Wales Emergency Department Data Collections; ICD-10, International Statistical Classification of Diseases and Related Health Problems 10th Revision; MH-AMB, Mental Health Ambulatory Collection; NDI, National Deaths Index; PHDAS, Pharmaceutical Drugs of Addiction System. 
methods to identify risk factors for adverse outcomes following initial opioid dispensing, comparing across opioids where relevant.

Each adverse outcome will be considered separately, with data censored at the earliest dataset conclusion or date of death (other than opioid-induced). Opioid-induced death will be considered as a competing risk for all non-death outcomes. We will investigate the effect of repeat incident exposure to prescribed opioids by using methods that incorporate multiple observations per person (eg, frailty models, generalised linear mixed models and generalised estimating equations).

Risk factors will be incorporated into multivariable models based on univariate analyses and retained if they show evidence of an association with the outcome. Risk factors will be identified for the entire cohort, adjusting for patient disease group. We will assess heterogeneity between disease groups in the effect of risk factors and exposures descriptively, by constructing models within each disease group, and formally through hypothesis testing of interaction terms.

Since we have individuals' linked hospital separations, we will account for time spent in hospital (and therefore time when the individual will not be using community-based prescriptions). The same applies for entry into opioid substitution therapy. These adjustments are strengths of our study and something that no previous analyses of opioid utilisation have undertaken.

\section{Aim 3: examining the impacts of opioid scheduling, regulation and} subsidy changes

We will analyse the potential impact of changes in opioid scheduling or subsidy. We summarise the approach using oxycodone as an example. For example, a tamper-resistant formulation (TRF) of CRO was PBS-subsidised on 1 April 2014. Existing studies of this change ${ }^{17} 76$ could not examine impact at the individual patient level. We will use interrupted time-series analysis to assess the impact of the introduction of TRF-CRO on dispensing of oxycodone and other opioids and rates of discontinuation and switching from CRO to another strong opioid, using April 2014 as the point of change. To account for seasonality in PBS dispensings, long-term trends and autocorrelation, we will use an autoregressive integrated moving average (ARIMA) approach. ${ }^{77}$ We will create ARIMA models for discontinuation, overall switching and switching from CRO to other opioids. Analyses will be stratified by indication for opioid prescribing, demographics and comorbid mental health and substance use problems. Tests will be adjusted for clustering, as some individuals could switch more than once.

\section{Sample size and power analysis}

Based on existing data on PBS dispensings, we will have $\sim 2$ million people initiating opioids during the study period, of whom over 400000 will have initiated onto oxycodone. We will have $\sim 13$ million person-years of follow-up. Our study will be powered to detect even small differences in risks of adverse outcomes. For a rare $(5 \%)$ exposure, we will have $90 \%$ power to detect an incidence rate ratio of 1.34 for an outcome with 2000 occurrences as statistically significant at 0.025 one-sided significance. Our ARIMA analyses will have more than $90 \%$ power to detect effect sizes of $1 .^{78}$ For example, preliminary analysis of $80 \mathrm{mg}$ CRO sales showed a mean of 13760 pack sales/month before TRF-CRO's introduction and an autoregressive term of $0.076 .{ }^{17}$ An effect size of 1 equates to a capacity to detect a drop of 962 pack sales/month.

\section{Methodological considerations}

There are several inherent limitations with each of the datasets. A key methodological consideration for this study is related to use of PBS data to define our cohort and assess exposure to prescribed opioids and other medicines. The PBS has two beneficiary types relating to the level of copayment for each medicine. General beneficiaries pay a general copayment amount, and concessional beneficiaries pay a lower concessional copayment amount. Until 2012, dispensings for PBS-listed medicines that attracted a government subsidy are captured. This occurs when the price of a medicine is above the PBS copayment threshold. ${ }^{30}$ All dispensings for concessional beneficiaries (approximately 25\% of Australians ${ }^{33}$ ) are captured, since all PBS medicines cost more than the copayment threshold. From April 2012 onwards, the Department of Human Services also maintains dispensing records for under copayment medicines. Of particular importance to this study is the lack of under copayment data in the PBS records prior to 2012; this has direct relevance as a number of PBS-listed opioids cost less than the general beneficiary copayment. ${ }^{45}$ To account for this issue, we will restrict some analyses to continuous cohorts of concessional beneficiaries so that we have complete ascertainment of their PBS medicines.

\section{ETHICS AND DISSEMINATION}

\section{Data storage, retention and access}

To protect privacy and confidentiality, approval for the linkage of health data is provided under strict conditions for the storage, retention and use of the data. The current approval permits storage of the data at one site, UNSW Sydney, for up to 7 years following the date of publication of results.

\section{Dissemination}

This will be the largest PMS study of prescribed opioids undertaken in Australia, linking exposure and outcomes and examining risk factors for adverse outcomes of prescribed opioids. It will also demonstrate the capacity of analysis of routinely collected data to inform about risks of opioid prescribing before problems develop. As such, this work has important translational promise, with direct relevance to regulatory authorities and agencies worldwide. It will provide evidence against which clinical guidelines in pain management can be evaluated. A key audience for dissemination will be policy and regulatory stakeholders (eg, PBAC), general 
practitioners, pain specialists and policy stakeholders in Australia and internationally. Project findings will be disseminated at scientific conferences and in peer-reviewed journals. We will also conduct targeted dissemination with policy makers, professional bodies and peak bodies in the pain, medicine and addiction fields (eg, via stakeholder workshops and advisory groups). As the study uses routinely collected health data, findings will be reported in accordance with the REporting of studies Conducted using Observational Routinely collected Data (RECORD) Statement. ${ }^{79}$

\section{Author affiliations}

${ }^{1}$ National Drug and Alcohol Research Centre, UNSW Sydney, Sydney, New South Wales, Australia

${ }^{2}$ Centre for Big Data Research in Health, UNSW Sydney, Sydney, New South Wales, Australia

${ }^{3}$ IMPACCT, Faculty of Health, University of Technology Sydney, Sydney, New South

Wales, Australia

${ }^{4}$ Concord Clinical School, The University of Sydney, Sydney, New South Wales, Australia

${ }^{5}$ School of Medicine and Public Health, Faculty of Health, The University of Newcastle, Newcastle, New South Wales, Australia

${ }^{6}$ Drug and Alcohol Clinical Services, Hunter New England, Newcastle, New South Wales, Australia

${ }^{7}$ Menzies Centre for Health Policy, Faculty of Medicine and Health, The University of Sydney, Sydney, New South Wales, Australia

Acknowledgements We wish to acknowledge our associate investigators for their contributions in developing the protocol for this study: Dr Andrea Schaffer, Dr Briony Larance, Dr Gabrielle Campbell, Dr Michael Coory, Professor Michael Farrell, Professor Milton Cohen, Professor Nicholas Buckley, Professor Paul Haber and Professor Rebecca Ivers.

Contributors All authors had involvement in developing the original protocol document upon which this manuscript was based. The study idea was conceived by NG, SAP, TD and LD. All authors provided input to the study design and developing the research questions and statistical analysis plan. NG and LD drafted the first iteration of the manuscript. All authors reviewed the manuscript and approved the final draft.

Funding This work is supported by a National Health and Medical Research Council Health (NHMRC) project grant (\#1138442). NG, SL and LD are supported by NHMRC research fellowships (\#1091878, \#1140938 and \#1135991). The National Drug and Alcohol Research Centre at UNSW Sydney is supported by funding from the Australian Government under the Substance Misuse Prevention and Service Improvements Grant Fund.

Competing interests The authors declare no direct competing interests relevant to this study protocol. Some of the authors have received investigator-initiated untied educational grants from Reckitt Benckiser/Indivior for studies of buprenorphinenaloxone (LD and RPM), buprenorphine depot (LD and AD), naloxone (SL and LD), the development of an opioid-related behaviour scale (LD and RPM) and a study of opioid substitution therapy uptake among chronic non-cancer pain patients (LD and RPM). Some of the authors have also received investigator-initiated untied educational grants for postmarketing surveillance studies of a tamper-resistant opioid formulation from Mundipharma (LD) and for tapentadol from Seqirus Pty Ltd (LD). No company had any knowledge or involvement in this study. AW is paid by the Australian Commonwealth government as the chair of the Pharmaceutical Benefits Advisory Committee (PBAC). SAP is a member of the Drug Utilisation SubCommittee (DUSC) of the PBAC.

Patient consent Not required.

Ethics approval The study protocol has received full ethical approval from the Australian Institute of Health and Welfare (AlHW) Ethics Committee (E02016/4/314), NSW Population and Health Services Research Committee (2017/HRE0208) and the ACT Health Human Research Ethics Committee (ETHLR.18.094).

Provenance and peer review Not commissioned; peer reviewed for ethical and funding approval prior to submission.
Open access This is an open access article distributed in accordance with the Creative Commons Attribution Non Commercial (CC BY-NC 4.0) license, which permits others to distribute, remix, adapt, build upon this work non-commercially, and license their derivative works on different terms, provided the original work is properly cited, appropriate credit is given, any changes made indicated, and the use is non-commercial. See: http://creativecommons.org/licenses/by-nc/4.0/.

\section{REFERENCES}

1. Pharmaceutical Benefits Division. Australian statistics on medicines 2015. Canberra: Commonwealth of Australia, 2016.

2. Blanch B, Pearson SA, Haber PS. An overview of the patterns of prescription opioid use, costs and related harms in Australia. $\mathrm{Br} J$ Clin Pharmacol 2014;78:1159-66.

3. Karanges EA, Blanch B, Buckley NA, et al. Twenty-five years of prescription opioid use in Australia: a whole-of-population analysis using pharmaceutical claims. Br J Clin Pharmacol 2016:82:255-67.

4. Beubler E, Jaksch W, Devulder J, et al. The White Paper on opioids and pain: a pan-European challenge: the European White Paper on the use of opioids in chronic pain management. J Pain Palliat Care Pharmacother 2006;20:79-87.

5. Furlan AD, Sandoval JA, Mailis-Gagnon A, et al. Opioids for chronic noncancer pain: a meta-analysis of effectiveness and side effects. CMAJ 2006;174:1589-94.

6. Manchikanti L, Ailinani $\mathrm{H}$, Koyyalagunta $\mathrm{D}$, et al. A systematic review of randomized trials of long-term opioid management for chronic non-cancer pain. Pain Physician 2011;14:91-121.

7. Degenhardt L, Black E, Breen C, et al. Trends in morphine prescriptions, illicit morphine use and associated harms among regular injecting drug users in Australia. Drug Alcohol Rev 2006;25:403-12.

8. Roxburgh A, Bruno R, Larance B, et al. Prescription of opioid analgesics and related harms in Australia. Med $J$ Aust 2011;195:280-4.

9. Roxburgh A, Burns L, Drummer OH, et al. Trends in fentany prescriptions and fentanyl-related mortality in Australia. Drug Alcohol Rev 2013;32:269-75

10. Roxburgh A, Hall WD, Burns L, et al. Trends and characteristics of accidental and intentional codeine overdose deaths in Australia. Med J Aust 2015;203:299.

11. Cicero TJ, Inciardi JA, Muñoz A. Trends in abuse of Oxycontin and other opioid analgesics in the United States: 2002-2004. J Pain 2005;6:662-72.

12. Katz N, Dart RC, Bailey E, et al. Tampering with prescription opioids: nature and extent of the problem, health consequences, and solutions. Am J Drug Alcohol Abuse 2011;37:205-17.

13. Compton WM, Volkow ND. Major increases in opioid analgesic abuse in the United States: concerns and strategies. Drug Alcohol Depend 2006;81:103-7.

14. General Accounting Office. Prescription drugs: Oxycontin abuse and diversion and efforts to address the problem (GAO-04-110). 2003. http://www.gao.gov/new.items/d04110.pdf

15. Campbell G, Nielsen S, Larance B, et al. Pharmaceutical opioid use and dependence among people living with chronic pain: associations observed within the Pain and Opioids in Treatment (POINT) Cohort. Pain Med 2015;16:1745-58.

16. Larance B, Lintzeris N, Bruno R, et al. The characteristics of a cohort who tamper with prescribed and diverted opioid medications. $J$ Subst Abuse Treat 2015;58:51-61.

17. Degenhardt L, Bruno R, Ali R, et al. The introduction of a potentially abuse deterrent oxycodone formulation: Early findings from the Australian National Opioid Medications Abuse Deterrence (NOMAD) study. Drug Alcohol Depend 2015;151:56-67.

18. Degenhardt L, Gisev N, Cama E, et al. The extent and correlates of community-based pharmaceutical opioid utilisation in Australia. Pharmacoepidemiol Drug Saf 2016;25:521-38.

19. Kassebaum NJ, Arora M, Barber RM, et al. Global, regional, and national disability-adjusted life-years (DALYs) for 315 diseases and injuries and healthy life expectancy (HALE), 1990-2015: a systematic analysis for the Global Burden of Disease Study 2015. Lancet 2016;388:1603-58.

20. Jones CM, Mack KA, Paulozzi LJ, et al. Pharmaceutical overdose deaths, United States, 2010. JAMA 2013;309:657-9.

21. Compton WM, Jones CM, Baldwin GT. Relationship between nonmedical prescription-opioid use and heroin use. $N$ Engl J Med 2016;374:154-63.

22. Frank RG, Pollack HA. Addressing the fentanyl threat to public health. N Engl J Med 2017;376:605-7.

23. Rudd RA, Seth $P$, David F, et al. Increases in drug and opioidinvolved overdose deaths - United States, 2010-2015. MMWR Morb Mortal Wkly Rep 2016;65:1445-52. 
24. Kochanek KD, Murphy SL, Xu J, et al. Deaths: final data for 2014. Natl Vital Stat Rep 2016;65:1-122.

25. Poudel DR, Acharya P, Ghimire S, et al. Burden of hospitalizations related to adverse drug events in the USA: a retrospective analysis from large inpatient database. Pharmacoepidemiol Drug Saf 2017;26:635-41.

26. Case A, Deaton A. Rising morbidity and mortality in midlife among white non-Hispanic Americans in the 21st century. Proc Natl Acad Sci U S A 2015;112:15078-83.

27. Dowell D, Haegerich TM, Chou R. CDC guideline for prescribing opioids for chronic pain - United States, 2016. JAMA 2016;65:1-49.

28. Center for Drug Evaluation and Research (CDER). Guidance for industry: abuse-deterrent opioids - evaluation and labeling. Rockville, MD: Food and Drug Administration, US Department of Health and Human Services, 2013.

29. Degenhardt L, Larance B, Peacock A, et al. Reducing extramedical use and harms of pharmaceutical opioids: the potential role of abuse-deterrent formulations. Lancet Psychiatry 2015;2:957-9.

30. Therapeutic Guidelines. eTG Complete: analgesic. Melbourne: Therapeutic Guidelines Ltd, 2018.

31. Blyth FM, March LM, Brnabic AJ, et al. Chronic pain in Australia: a prevalence study. Pain 2001;89:127-34.

32. Noble M, Treadwell JR, Tregear SJ, et al. Long-term opioid management for chronic noncancer pain. Cochrane Database Syst Rev 2010:CD006605.

33. Roxburgh A, Degenhardt L, Burns L, et al. Opioid-related mortality in Australia. Drug Alcohol Rev 2014;33:7.

34. Gisev N, Pearson SA, Blanch B, et al. Initiation of strong prescription opioids in Australia: cohort characteristics and factors associated with the type of opioid initiated. $\mathrm{Br} J$ Clin Pharmacol 2016;82:1123-33.

35. Campbell G, Bruno R, Lintzeris N, et al. Defining problematic pharmaceutical opioid use among people prescribed opioids for chronic noncancer pain: do different measures identify the same patients? Pain 2016;157:1489-98.

36. Campbell G, Nielsen S, Bruno R, et al. The Pain and Opioids IN Treatment study: characteristics of a cohort using opioids to manage chronic non-cancer pain. Pain 2015;156:231-42.

37. Clarke H, Soneji N, Ko DT, et al. Rates and risk factors for prolonged opioid use after major surgery: population based cohort study. BMJ 2014;348:g1251.

38. Cochran BN, Flentje A, Heck NC, et al. Factors predicting development of opioid use disorders among individuals who receive an initial opioid prescription: mathematical modeling using a database of commercially-insured individuals. Drug Alcohol Depend 2014;138:202-8.

39. Rogers KD, Kemp A, McLachlan AJ, et al. Adverse selection? A multi-dimensional profile of people dispensed opioid analgesics for persistent non-cancer pain. PLoS One 2013;8:e80095.

40. Sullivan MD. Who gets high-dose opioid therapy for chronic noncancer pain? Pain 2010;151:567-8.

41. World Health Organization. The importance of pharmacovigilance: safety monitoring of medicinal products. Geneva: WHO, 2002.

42. Coste J. Diverging approaches of pharmacovigilance and pharmacoepidemiology to assessing drug safety: epistemological and ethical implications. Pharmacoepidemiol Drug Saf 2017;26:600-2

43. Califf RM, Woodcock J, Ostroff S. A proactive response to prescription opioid abuse. N Engl J Med 2016;374:1480-5.

44. International Association for the Study of Pain. IASP statement on opioids, 2018. http://www.iasp-pain.org/Advocacy/Content.aspx? ItemNumber $=7194$

45. Mellish L, Karanges EA, Litchfield MJ, et al. The Australian Pharmaceutical Benefits Scheme data collection: a practical guide for researchers. BMC Res Notes 2015;8:634.

46. World Health Organization Collaborating Centre for Drugs Statistics Methodology. ATC/DDD Index 2018, 2017. http://www.whocc.no/ atc_ddd_index/ (Accessed 3 Aug 2018).

47. Gisev N, Pearson SA, Karanges EA, et al. To what extent do data from pharmaceutical claims under-estimate opioid analgesic utilisation in Australia? Pharmacoepidemiol Drug Saf 2018;27:550-5.

48. Degenhardt L, Blanch B, Gisev N, et al. The POPPY Research Programme protocol: investigating opioid utilisation, costs and patterns of extramedical use in Australia. BMJ Open 2015;5:e007030.

49. Blanch B, Degenhardt L, Buckley NA, et al. Prescription opioid access patterns and factors associated with increasing number of prescribers, pharmacies, and dispensings: an observational study using pharmaceutical claims. Pain Med 2018;19:1170-83.

50. Blanch B, Daniels B, Litchfield M, et al. Looking forward and looking back: the balancing act in new drug user designs for pharmacoepidemiological research. Pharmacoepidemiol Drug Saf 2015;24:1117-9.

51. Spilsbury K, Rosman D, Alan J, et al. Cross border hospital use: analysis using data linkage across four Australian states. Med J Aust 2015;202:582-6.

52. Nielsen S, Degenhardt L, Hoban B, et al. A synthesis of ora morphine equivalents (OME) for opioid utilisation studies. Pharmacoepidemiol Drug Saf 2016;25:733-7.

53. MIMS Australia. MIMS online: drug interactions. MIMS Australia: St Leonards, 2014

54. Australian Medicines Handbook. Australian medicines handbook. Adelaide: Australian Medicines Handbook Pty Ltd, 2014.

55. In: Baxter K, Preston CL, eds. Stockley's drug interactions. 10th edn. London: edPharmaceutical Press, 2015.

56. Ringland C, Mant A, McGettigan P, et al. Uncovering the potential risk of serotonin toxicity in Australian veterans using pharmaceutical claims data. Br J Clin Pharmacol 2008;66:682-8.

57. Larance B, Degenhardt L, Lintzeris N, et al. Definitions related to the use of pharmaceutical opioids: extramedical use, diversion, nonadherence and aberrant medication-related behaviours. Drug Alcohol Rev 2011;30:236-45.

58. Bachs LC, Bramness JG, Engeland A, et al. Repeated dispensing of codeine is associated with high consumption of benzodiazepines. Nor Epidemiol 2008;18:185-90.

59. Logan J, Liu Y, Paulozzi L, et al. Opioid prescribing in emergency departments: the prevalence of potentially inappropriate prescribing and misuse. Med Care 2013;51:646-53.

60. White AG, Birnbaum HG, Schiller M, et al. Analytic models to identify patients at risk for prescription opioid abuse. Am J Manag Care 2009;15:897-906.

61. Bramness JG, Furu K, Engeland A, et al. Carisoprodol use and abuse in Norway: a pharmacoepidemiological study. Br J Clin Pharmacol 2007;64:210-8.

62. Frauger $\mathrm{E}$, Pauly V, Thirion X, et al. Estimation of clonazepam abuse liability: a new method using a reimbursed drug database. Int Clin Psychopharmacol 2009;24:318-24.

63. Frauger E, Pauly V, Natali F, et al. Patterns of methylphenidate use and assessment of its abuse and diversion in two French administrative areas using a proxy of deviant behaviour determined from a reimbursement database: main trends from 2005 to 2008. CNS Drugs 2011;25:415-24.

64. Wilsey BL, Fishman SM, Gilson AM, et al. Profiling multiple provider prescribing of opioids, benzodiazepines, stimulants, and anorectics. Drug Alcohol Depend 2010;112:99-106.

65. Lozano R, Naghavi M, Foreman K, et al. Global and regional mortality from 235 causes of death for 20 age groups in 1990 and 2010: a systematic analysis for the Global Burden of Disease Study 2010 Lancet 2012;380:2095-128.

66. Cepeda MS, Fife D, Chow W, et al. Opioid shopping behavior: how often, how soon, which drugs, and what payment method. J Clin Pharmacol 2013;53:112-7.

67. Cepeda MS, Fife D, Yuan Y, et al. Distance traveled and frequency of interstate opioid dispensing in opioid shoppers and nonshoppers. J Pain 2013;14:1158-61.

68. Cepeda MS, Fife D, Chow W, et al. Assessing opioid shopping behaviour: a large cohort study from a medication dispensing database in the US. Drug Saf 2012;35:325-34.

69. Peirce GL, Smith MJ, Abate MA, et al. Doctor and pharmacy shopping for controlled substances. Med Care 2012;50:494-500.

70. Pradel V, Thirion X, Ronfle E, et al. Assessment of doctor-shopping for high dosage buprenorphine maintenance treatment in a French region: development of a new method for prescription database. Pharmacoepidemiol Drug Saf 2004;13:473-81.

71. Martyres RF, Clode D, Burns JM. Seeking drugs or seeking help? Escalating "doctor shopping" by young heroin users before fatal overdose. Med J Aust 2004;180:211-4.

72. Braker LS, Reese AE, Card RO, et al. Screening for potential prescription opioid misuse in a michigan medicaid population. Fam Med 2009;41:729-34.

73. Katz N, Panas L, Kim M, et al. Usefulness of prescription monitoring programs for surveillance--analysis of Schedule II opioid prescription data in Massachusetts, 1996-2006. Pharmacoepidemiol Drug Saf 2010;19:115-23.

74. Rice JB, White AG, Birnbaum HG, et al. A model to identify patients at risk for prescription opioid abuse, dependence, and misuse. Pain Med 2012:13:1162-73.

75. Seal KH, Shi Y, Cohen G, et al. Association of mental health disorders with prescription opioids and high-risk opioid use in US veterans of Iraq and Afghanistan. JAMA 2012;307:940-7.

76. Larance B, Dobbins T, Peacock A, et al. The effect of a potentially tamper-resistant oxycodone formulation on opioid use and harm: 
main findings of the National Opioid Medications Abuse Deterrence (NOMAD) study. Lancet Psychiatry 2018;5:155-66.

77. Box GE, Jenkins GM, Reinsel GC, et al. Time series analysis: forecasting and control. New Jersey: John Wiley \& Sons, 2015.

78. Zhang F, Wagner AK, Ross-Degnan D. Simulation-based power calculation for designing interrupted time series analyses of health policy interventions. J Clin Epidemiol 2011;64:1252-61.

79. Benchimol El, Smeeth L, Guttmann A, et al. The REporting of studies Conducted using Observational Routinely-collected health Data (RECORD) statement. PLoS Med 2015;12:e1001885.

80. Australian Bureau of Statistics. 2033.0.55.001 - Census of population and housing: Socio-Economic Indexes for Areas (SEIFA), Australia, 2011, 2013. http://www.abs.gov.au/AUSSTATS/abs@.nsf/DetailsPage/ 2033.0.55.0012011? OpenDocument (Accessed 3 Aug 2018).

81. Australian Bureau of Statistics. 1216.0.15.003 - Australian Standard Geographical Classification (ASGC) remoteness area correspondences, 2006, 2007. http://www.abs.gov.au/
AUSSTATS/abs@.nsf/Lookup/1216.0.15.003Main+Features12006? OpenDocument (Accessed 3 Aug 2018).

82. World Health Organization. International Statistical Classification of Diseases and Related Health Problems 10th Revision (ICD-10), 2016. http://apps.who.int/classifications/icd10/browse/2015/en\#/C80.9 (Accessed 3 Aug 2018).

83. World Health Organization. International Classification of Diseases for Oncology (ICD-O-3). $2013 \mathrm{http}: / /$ codes.iarc.fr/.

84. Charlson M, Szatrowski TP, Peterson J, et al. Validation of a combined comorbidity index. J Clin Epidemiol 1994;47:1245-51.

85. Sloan KL, Sales AE, Liu CF, et al. Construction and characteristics of the RxRisk-V: a VA-adapted pharmacy-based case-mix instrument. Med Care 2003;41:761-74.

86. Pratt NL, Kerr M, Barratt JD, et al. The validity of the Rx-Risk Comorbidity Index using medicines mapped to the Anatomical Therapeutic Chemical (ATC) Classification System. BMJ Open 2018;8:e021122. 